\title{
COMPETENCIA CHINA: BUENA, VARIADA Y BARATA*
}

\author{
Sebastián Claro y Ramón Delpiano
}

\begin{abstract}
Las importaciones chinas en Chile crecieron a una tasa promedio anual de 29\% entre 1990 y 2005, mientras que las importaciones del resto del mundo lo hicieron a una tasa promedio de 9,6\%. En este trabajo se concluye que el principal determinante del fuerte incremento en la participación de China es un aumento en la calidad relativa de los productos chinos, que eleva la demanda por variedades chinas así como el número de variedades disponibles desde China. La caída en el precio relativo de los productos chinos explica un porcentaje menor de la penetración de China. Los resultados sugieren, a su vez, que los incrementos en la calidad de los productos chinos se deben a aumentos en la productividad de las empresas de ese país. Esto resalta la necesidad de fomentar mejoras en productividad y elevar sustancialmente la calidad de la educación en
\end{abstract}

Sebastián Claro. Ingeniero Comercial y Magíster en Economía, Pontificia Universidad Católica de Chile. Ph.D. en Economía, Universidad de California, Los Angeles. Profesor, Instituto de Economía, Pontificia Universidad Católica de Chile. Email: sclaro@faceapuc.cl.

Ramón Delpiano. Ingeniero Comercial, Pontificia Universidad Católica de Chile. Investigador, Fundación Jaime Guzmán. Email: rdelpiano@fjguzman.cl.

* Agradecemos los comentarios y sugerencias de Roberto Álvarez, Juan Carlos Hallak y un árbitro anónimo de Estudios Públicos. Sebastián Claro agradece el apoyo financiero de Fundación Andes a través del Proyecto C-140609. 
Chile. De otra manera — señalan los autores de este estudio—-, la capacidad de diferenciarse de los productos chinos con una baja relación precio/calidad es muy limitada, lo que podría llevar a la economía hacia una mayor especialización en bienes intensivos en recursos naturales, donde las ventajas comparativas son evidentes.

\section{Introducción}

L

penetración de productos chinos en los mercados mundiales en los últimos 15 años es impresionante. Las exportaciones totales de China pasaron de US\$ 62,9 billones en 1990 a US\$ 762 billones el 2005, lo que representa una tasa de crecimiento promedio anual de $18 \%$, más del doble del crecimiento del producto chino en ese período. Chile no ha estado ausente de este fenómeno: en el mismo período la tasa de crecimiento promedio anual de las importaciones chilenas desde China fue de $29 \%$, mientras que el resto de las importaciones creció a una tasa promedio del 9,6\%. De esta manera, China pasó de representar menos de un $1 \%$ de las importaciones en 1990 a representar un 8,6\% de las importaciones totales de Chile el 2005.

La mayor disponibilidad de productos chinos a bajo costo tiene sin duda beneficios para los consumidores. Sin embargo, es evidente que la mayor competencia de productos chinos tiene también importantes consecuencias sobre los productores. En parte, el debate sobre globalización está fuertemente influenciado por el impacto que tiene la apertura de países grandes y abundantes en mano de obra — como China — sobre la industria en el resto de los países. Por una parte hay quienes auguran un permanente desplazamiento de la industria manufacturera mundial hacia China debido a los menores costos de producción y a la caída en los costos de transporte. Lo anterior generaría un desplazamiento de la producción en países desarrollados hacia bienes o servicios intensivos en capital humano (ideas) o hacia productos intensivos en recursos naturales en países como Chile. Alternativamente, la literatura ha enfatizado la posibilidad de diferenciar los productos manufactureros en términos de calidad. Así, los países podrían escapar a la competencia de China y aprovechar su oferta de productos a bajos precios cambiando su mix de productos hacia una canasta de mejor cali$\mathrm{dad}^{1}$. Estos dos escenarios tienen consecuencias muy distintas en los pa-

${ }^{1}$ Véase Leamer (2006) y Bernard et al. (2006). Véanse también los artículos de Abreu (2006), Arellano (2006), Claro (2006a) y Galperín et al. (2006) sobre el impacto de la competencia china en distintos países de América Latina. Resulta interesante 
trones de producción y especialización de los países². Es crucial, por lo tanto, determinar en qué medida la diferenciación por calidad es un determinante relevante de los patrones de especialización y comercio, así como identificar condiciones bajo las cuales esta diferenciación es posible.

Este trabajo constituye un primer paso para responder estas interrogantes. Se presenta un análisis detallado de las importaciones de Chile entre 1990 y 2005 de manera de identificar y cuantificar los determinantes del mayor crecimiento de las importaciones desde China en relación a las importaciones desde el resto del mundo. Para ello, usamos datos de importaciones clasificadas a nivel de 8 dígitos del Sistema Armonizado obtenidos en el Servicio Nacional de Aduanas. Estos datos permiten cuantificar con alto nivel de precisión la penetración de China en distintas categorías de productos, pudiendo descomponer la mayor tasa de crecimiento de las importaciones desde China en tres componentes: a) una caída en el precio relativo de los productos chinos, b) un aumento en el número de variedades o productos disponibles desde China, y c) un aumento en la calidad relativa de los productos de China vis-à-vis el resto del mundo. Conceptualmente, diferencias en las cantidades importadas (quantum) de productos similares desde distintos países que no se explican por diferencias de precios, se deben a diferencias en la calidad de los productos o diferencias en el número de variedades disponibles en cada país ${ }^{3}$.

El análisis empírico entrega tres resultados importantes. Primero, los productos chinos son en promedio un $45 \%$ más baratos que los productos del resto del mundo, y esta diferencia aumenta entre 1990 y 2005 a una tasa anual del $0,7 \%$. Sin embargo, la caída en el precio relativo de los productos chinos sólo explica un porcentaje menor del aumento en la penetración de

notar que en México y Brasil la competencia china no sólo afecta a los productores que sustituyen importaciones sino que también impacta fuertemente en los mercados de exportaciones debido a la similitud de la canasta exportadora. Para mayores detalles del caso mexicano se recomienda leer a Dussel y Dong (2004) y a Hanson y Robertson (2006).

${ }^{2}$ Una implicancia adicional de la especialización en bienes intensivos en mano de obra es el posible incremento en la desigualdad salarial. En la medida que la competencia de China se traduzca en una caída en el precio relativo de bienes intensivos en mano de obra menos calificada, el retorno relativo a estos factores cae (Beyer et al., 2000). En la medida que un país no produzca los bienes cuyo precio relativo cae dada la mayor oferta de China, se aísla a los trabajadores menos calificados de las presiones a la baja en sus salarios, y se producen incrementos en retornos reales para todos los factores.

${ }^{3}$ La literatura generalmente ha usado diferencias en precios unitarios de productos como proxies de diferencias en calidad (Schott, 2004). Nuestra metodología atribuye a diferencias en calidad la parte de las diferencias en consumo que no son explicadas por diferencias de precios o número de variedades disponibles. 
China. Esto nos lleva al segundo resultado: la principal causa de la alta penetración de productos chinos es un aumento en su calidad relativa a la del resto del mundo. La mayor calidad genera no sólo un mayor consumo de variedades chinas, sino que también eleva el número de variedades producidas en China. Esto es, las empresas (países) que producen bienes de mayor calidad no sólo venden más por variedad producida sino que también ofrecen un mayor número de variedades ${ }^{4}$. Finalmente, los resultados muestran que el aumento en la calidad relativa de los productos chinos está asociada con mejoras en productividad. Así, los incrementos en productividad de los productores chinos han permitido mantener precios bajos aún con alzas significativas en los precios de los factores. Aunque no es el objetivo de este trabajo analizar las causas de los cambios en productividad de las empresas chinas, existe evidencia de que la mayor penetración de empresas extranjeras está asociada con una mayor productividad de las empresas domésticas (Claro, 2006b) $)^{5}$.

En general, los resultados sugieren que la diferenciación por calidad es posible, pero que requiere de importantes mejoras en productividad. Para un país como Chile, las políticas que generan mejoras en productividad pueden tomar variadas formas. Por una parte se requiere facilitar el proceso de creación /destrucción de empresas. La productividad aumenta en la medida que se incentive la creatividad y el desarrollo e implementación de nuevas ideas, para lo que es necesario una mayor facilidad en la creación de empresas, flexibilidad en su funcionamiento, estabilidad en las reglas del juego y pocas restricciones para cerrar la empresa en la medida que su capacidad de generar valor sea baja. Otro aspecto relevante para mejorar la productividad es desarrollar políticas serias que mejoren significativamente la calidad de la educación. Este último punto es muy relevante. Una fuerza de trabajo más educada no sólo es capaz de asimilar mejor las ideas/tecnologías más modernas y novedosas, sino que en sí constituye una fuente de mayor productividad. La lentitud de un proceso de reforma educacional profundo hace más difícil encontrar los nichos para diferenciarse de la avalancha china, incrementando la competencia en productos manufactureros y empujando la producción hacia sectores con evidentes ventajas comparativas, como los intensivos en recursos naturales. Esta solución no es necesariamente perjudicial desde el punto de vista de bienestar agregado, aun cuando los problemas distributivos asociados con una alta dependencia de

${ }^{4}$ Véase Brambilla (2006) para una conclusión similar.

${ }^{5}$ Adams et al. (2004) y Rodrik (2006) desarrollan hipótesis similares para explicar la alta sofisticación de las exportaciones chinas comparadas con las de países con similar ingreso per cápita. Véase también Schott (2006). 
los recursos naturales pueden ser altos y la capacidad de crecimiento en el largo plazo limitada ${ }^{6}$. En todo caso, para evitar la especialización en recursos naturales se requiere de una alta productividad en otros bienes y servicios transables y de un capital humano que permita ofrecer bienes y servicios de alta calidad.

El resto del trabajo se divide de la siguiente manera. La sección 2 describe los datos usados y la sección 3 presenta una descomposición de las importaciones de China y del resto del mundo en distintos márgenes. La sección 4 desarrolla un modelo simple para estimar la calidad de los productos chinos, y las estimaciones empíricas se presentan en la sección 5. La sección 6 discute algunas conclusiones.

\section{Datos}

Los datos usados son todas las partidas de importación de aduanas entre 1990 y 2005 clasificadas en categorías de 8 dígitos del Sistema Armonizado. Cada observación contiene el valor CIF y la cantidad (en unidades) de las importaciones de cada país en cada año para cada partida arancelaria. Ejemplos de categorías de 8 dígitos incluyen kilos de sales y esteres de ácido cítrico (3918.1500), número de camisones y pijamas de algodón para hombres (6207.2100), o número de micrófonos (8518.1000). Para efectos del trabajo, agrupamos los países en dos categorías: importaciones desde China — con subíndice $c$ - e importaciones agregadas desde el resto de los países en la categoría Resto del Mundo, identificada con el subíndice $r$.

El Cuadro $\mathrm{N}^{0} 1$ muestra las importaciones totales de Chile desde $c$ y $r$ en dólares corrientes entre 1990 y 2005. Los productos importados (definidos como categorías de 8 dígitos) se dividen en 3 grupos: aquellos importados exclusivamente desde $c$, aquellos importados exclusivamente desde $r$, y aquellos productos importados desde ambos orígenes (categorías comunes). Las importaciones totales difieren de las importaciones totales declaradas en los informes del Banco Central por tres razones. Primero, las importaciones por motivos militares no son declaradas en aduana y sí están incorporadas en los datos del Banco Central. Segundo, productos que entran a Chile a través de zonas francas y que son re-exportados a terceros países son incluidos en las estadísticas del Banco Central pero son excluidos de los datos del Servicio Nacional de Aduanas, que sólo incluye las

${ }^{6}$ Véase Leamer et al. (1999) para una discusión de la relación entre abundancia en recursos naturales y distribución del ingreso. Véase Sachs y Warner (1997), Manzano y Rigobon (2001), y Lederman y Maloney (2002). 


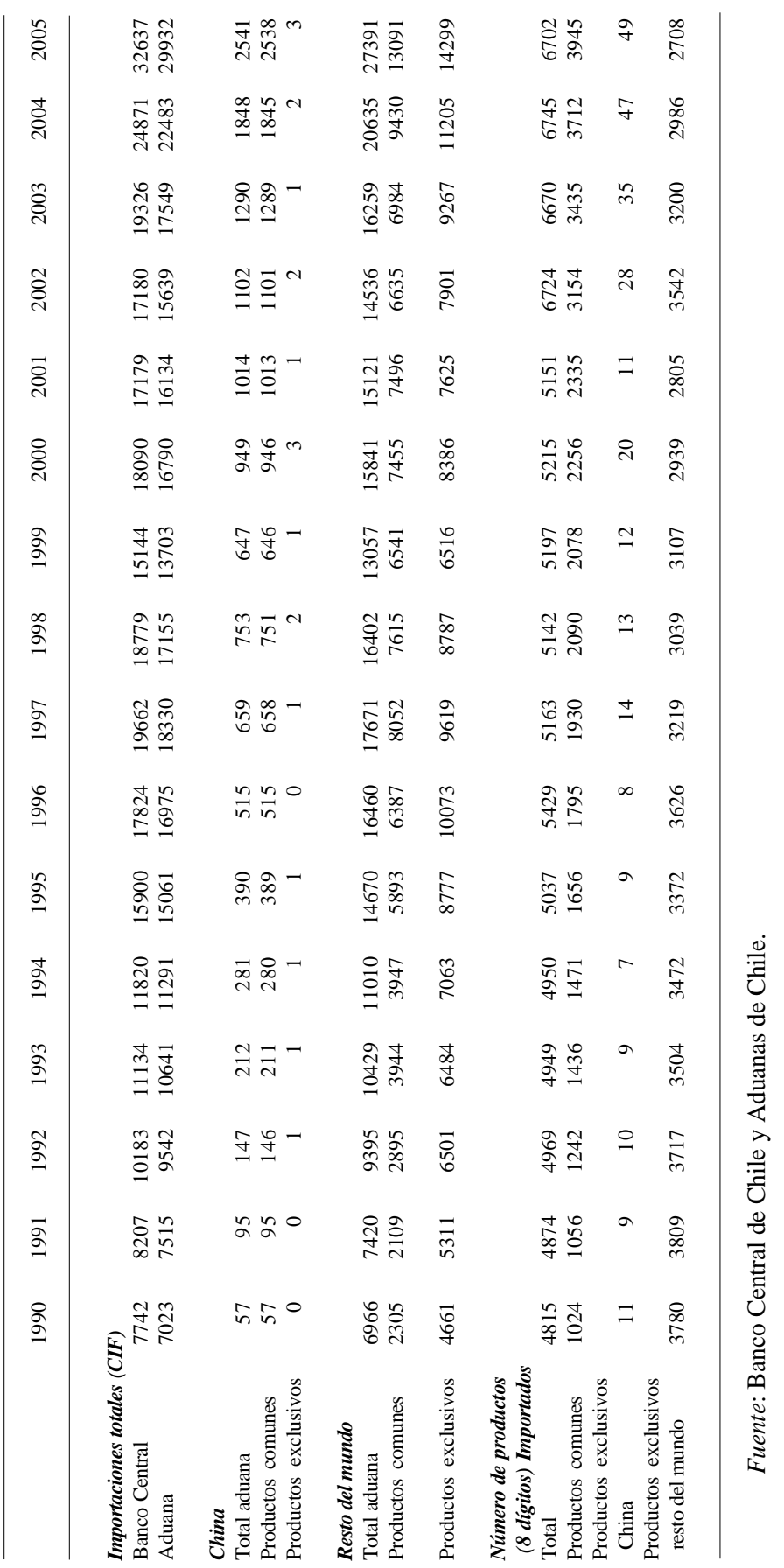


importaciones de zonas francas que tienen como destino final el mercado chileno. Finalmente, las adquisiciones de barcos chilenos en el exterior (como combustible) tampoco son contabilizadas en aduana. En nuestro trabajo usamos los datos de importaciones reportados por Aduanas.

Las importaciones desde China representaban un $0,8 \%$ del total de importaciones en 1990, mientras que el 2005 representaban un 8,6\% del total. En términos absolutos, las importaciones desde China pasaron de 57 a 2.540 millones de dólares en el período, lo que representa una tasa de crecimiento promedio anual cercana a $29 \%$. La tasa de crecimiento promedio de las importaciones desde el resto del mundo entre 1990 y 2005 fue de 9,6\%. La parte inferior del Cuadro $\mathrm{N}^{\circ} 1$ presenta el número de categorías importadas desde $c$ y $r$. Por ejemplo, en 2005 Chile importó 6.702 productos definidos a 8 dígitos. Del total, 3.945 eran comunes entre China y el resto del mundo, 49 eran exclusivos de China y 2.708 eran exclusivos del resto del mundo ${ }^{7}$.

\section{Metodología}

Basados en el trabajo de Hummels y Klenow (2005), definimos la participación de China en las importaciones en la industria $i$ en el período $t$ como la razón entre las importaciones de $c$ y $r$ :

$$
S_{i t}=\frac{M_{c i t}}{M_{r i t}}=\frac{\sum_{j \in N_{c i t}} M_{c j t}}{\sum_{j \in N_{r i t}} M_{r j t}},
$$

donde $M_{c i t}$ es el valor de las importaciones de $c$ en la industria $i$ el año $t$, que es igual a la suma de las importaciones desde $c$ en todos los productos $j$ (definidos a 8 dígitos) que pertenecen a la industria $i$. El índice de participación $S_{t}$ puede ser calculado en distintos niveles de agregación $i$, esto es, 8 dígitos, 2 dígitos, 1 dígito. En el nivel más agregado (1 dígito), $S$ mide la razón de las importaciones totales desde China y el resto del mundo. En el nivel más desagregado (8 dígitos), $S$ mide la razón del valor de importaciones de China y el resto del mundo en cada producto $j$. Usando los datos del Cuadro $\mathrm{N}^{\circ} 1$, el Cuadro $\mathrm{N}^{\circ} 2$ muestra el índice de participación agregado de China, que pasó de 0,8\% en 1990 a 9,3\% en 2005. Para Estados Unidos,

${ }^{7}$ Existen algunas categorías en las cuales Aduanas reporta valores de importación (CIF) positivos, pero cantidades iguales a cero. Estas categorías —que representan un porcentaje ínfimo de las importaciones totales- fueron eliminadas del análisis.

${ }^{8}$ A nivel agregado, la participación de China en las importaciones totales de Chile es $S /(1+S)$. 


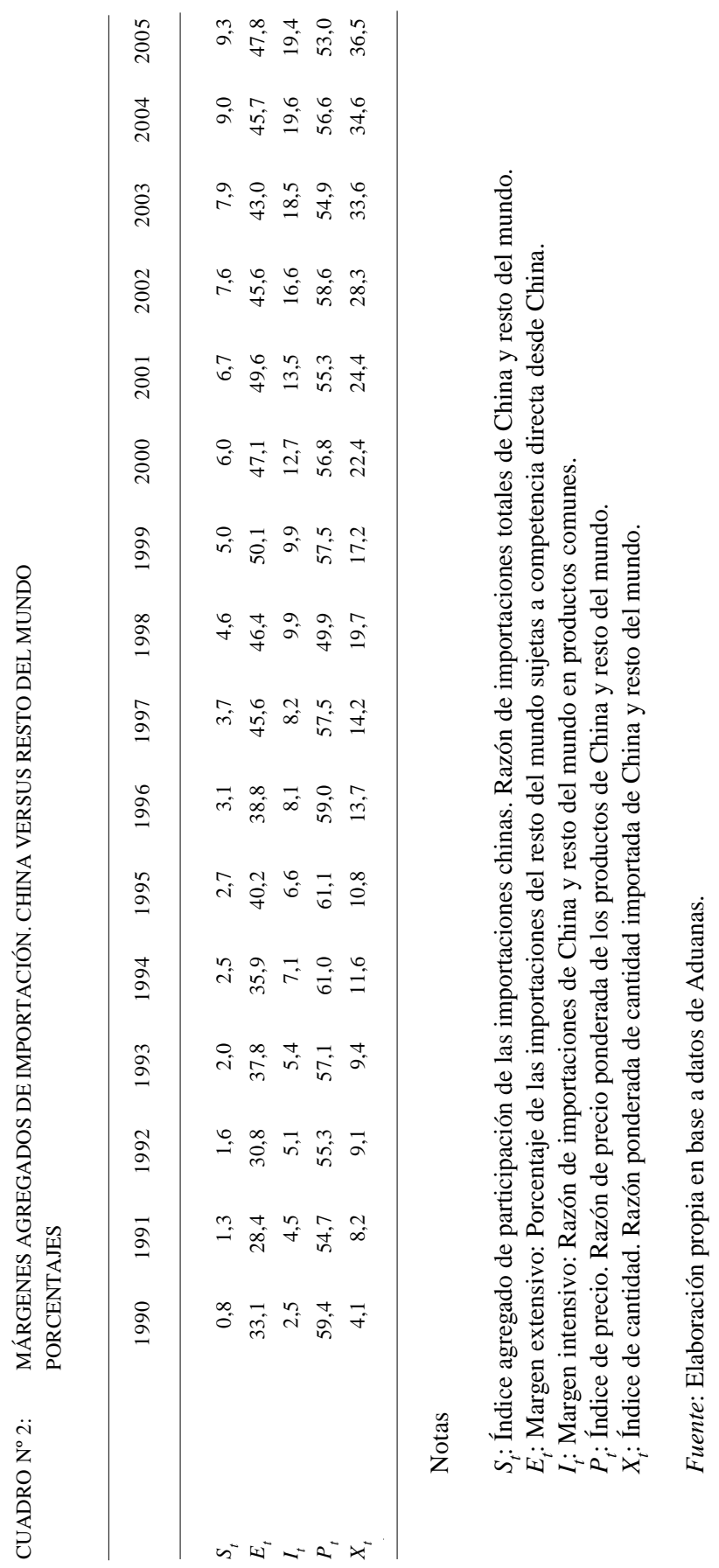


Hummels y Klenow (2005) calculan el índice de participación agregado de China en 1995 en 9,3\%.

El índice de participación puede ser expresado como el producto de un margen extensivo y un margen intensivo:

$$
S_{i t}=\frac{\sum_{j \in N_{c i t}} M_{c j t}}{\sum_{j \in N_{r i t}} M_{r j t}}=\frac{\sum_{j \in N_{c i t}} M_{r j t}}{\sum_{j \in N_{r i t}} M_{r j t}} \cdot \frac{\sum_{j \in N_{c i t}} M_{c j t}}{\sum_{j \in N_{c i t}} M_{r j t}}=E_{i t} \cdot I_{i t}
$$

El margen extensivo mide el porcentaje de las importaciones de $r$ que están sujetas a competencia directa de productos chinos, esto es, el porcentaje de las importaciones de $r$ en productos comunes con China. El margen intensivo compara las importaciones de $c$ y $r$ en un conjunto de productos comunes. Así, mide la "intensidad" de la competencia entre China y el resto del mundo en categorías comunes. El Cuadro $\mathrm{N}^{\circ} 2$ presenta los cálculos para el margen extensivo y el margen intensivo a nivel agregado. El año 2005, un $47,8 \%$ de las importaciones del resto del mundo estaban sujetas a competencia directa desde China, y el valor de las importaciones desde China era un 19,4\% del valor de las importaciones desde el resto del mundo en productos comunes. La Figura $\mathrm{N}^{\mathrm{o}} 1$ muestra $S_{t}, E_{t}$ e $I_{t}$ entre 1990 y 2005.

FIGURA N ${ }^{\circ}$ 1: EVOLUCIÓN DEL ÍNDICE DE PARTICIPACIÓN AGREGADA, MARGEN INTENSIVO Y MARGEN EXTENSIVO. CHINA VERSUS RESTO DEL MUNDO: 1990-2005

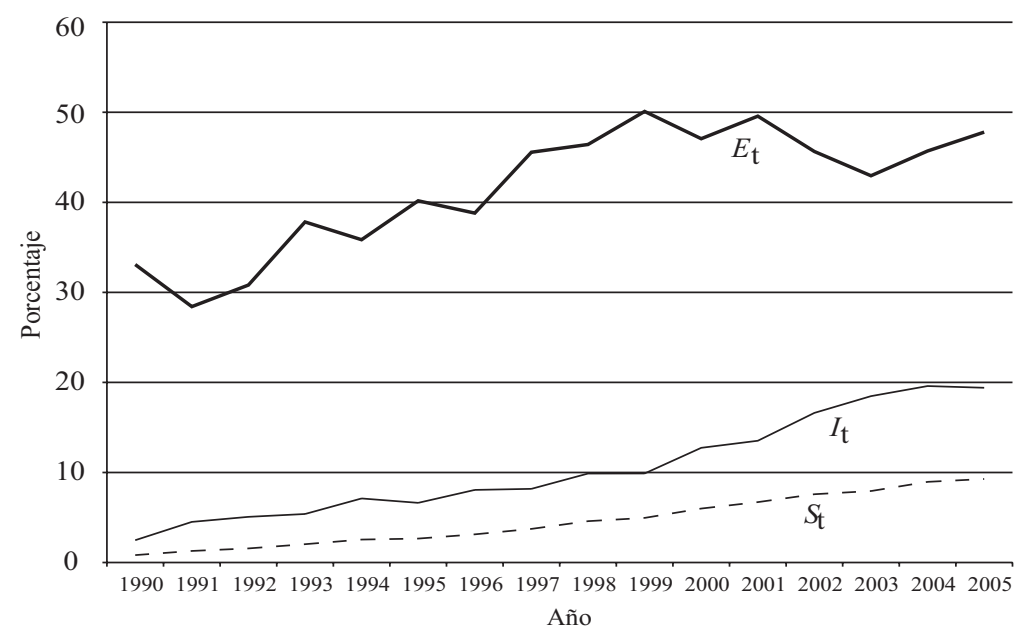


Se observan dos períodos diferentes. Entre 1990 y 1999 el índice de participación $S_{t}$ crece tanto por un aumento en el margen extensivo como en el margen intensivo. A partir de 1999 se observa un quiebre en la tendencia del margen extensivo, que permanece relativamente constante en un nivel cercano a $50 \%$, mientras que el margen intensivo mantiene una tasa de crecimiento constante. En otras palabras, el mayor crecimiento de las importaciones desde China — que es evidente en el sostenido crecimiento del índice agregado de participación $S_{t}$ - se explica por un aumento sostenido en el porcentaje de productos importados desde China —especialmente en los 1990 — así como por un crecimiento en la participación de mercado de China en productos comunes con $r$.

El margen intensivo puede a su vez ser expresado como el producto de un índice de precios y de un índice de cantidad. Intuitivamente, al nivel más desagregado la razón del valor de las importaciones entre $c$ y $r$ es el producto de la razón de precios unitarios y la razón de cantidades importadas. A mayores niveles de agregación, la razón de importaciones en categorías comunes puede expresarse como ${ }^{9}$ :

$$
I_{i t}=P_{i t} \cdot X_{i t}
$$

donde $P_{i t}=\prod_{j \in N_{c i t}}\left(p_{c j t} / p_{r j t}\right)^{\omega_{j t}}$ y $X_{i t}=\prod_{j \in N_{c i t}}\left(X_{c j t} / X_{r j t}\right)^{\omega_{j t}}$ son índices de precio y cantidad respectivamente. $p_{k j t}$ es el precio unitario de importación del producto $j$ proveniente del país $k=c, r$ el año $t$ calculado como $p_{k j t}=M_{k j t} / X_{k j t}$, donde $X_{k j t}$ es la cantidad importada del producto $j$ desde $k$. El ponderador $\omega_{j t}$ es la media logarítmica de la participación del producto $j$ en las importaciones de $c$ y en la importaciones de $r$ en aquellas categorías comunes con $c^{10}$. En otras palabras, $P_{i t}$ es un promedio ponderado de la razón de precios entre China y el resto del mundo en categorías comunes —donde $p_{c j t} / p_{r j t}$ puede ser calculado— que valora más aquellos productos que representan una proporción mayor de las importaciones en categorías comunes. Intuitivamente, un margen intensivo menor que 1 -como es el caso de China - puede reflejar menores precios de productos chinos sin mayores diferencias en cantidades consumidas, o puede reflejar diferencias en cantidades importadas con precios similares, o una combinación de am-

${ }^{9}$ Véase Sato (1976), Feenstra (1994) y Broda y Weinstein (2006).

${ }^{10} \omega_{j}=\left(\frac{S_{j c}-S_{j r}}{\ln S_{j c}-\ln S_{j r}}\right) /\left(\sum_{j} \frac{S_{j c}-S_{j r}}{\ln S_{j c}-1 \mathrm{n} S_{j r}}\right)$ donde $S_{j n}=p_{j n}^{k} x_{j n}^{k} / \sum_{j} p_{j n}^{k} x_{j n}^{k}$ 
bas. El Cuadro $\mathrm{N}^{\mathrm{o}} 2$ reporta los índices de precio y cantidad calculados a nivel agregado, y la Figura $N^{\circ} 2$ muestra $I, P_{t}$ y $X_{t}$ para todo el período. En promedio, los productos chinos tienen un valor unitario entre $50 \%$ y $60 \%$ del valor del resto del mundo, lo que es similar al 56\% estimado por Hummels and Klenow (2004) para Estados Unidos en 1995. Se observa una tendencia decreciente pero marginal en $P_{t}$-la tasa de crecimiento promedio anual es $-0,7 \%$ - lo que refleja un pequeño abaratamiento de los productos chinos ${ }^{11}$. En cambio, las cantidades consumidas de productos chinos aumentan fuertemente. El índice de cantidad pasa de un 4,1\% en 1990 a un $36,5 \%$ el año 2005, lo que muestra que las categorías comunes de las importaciones desde China han crecido en cantidad a una tasa anual 15,6 puntos porcentuales mayor que las importaciones desde el resto del mundo.

Dos elementos llaman la atención del Cuadro $\mathrm{N}^{\circ}$ 2. Por una parte, el consumo de productos chinos es bajo comparado con productos similares del resto del mundo (misma categoría 8 dígitos) tomando en cuenta su menor costo. ¿Qué explica que en promedio la cantidad consumida de produc-

FIGURA N ${ }^{\circ}$ 2: $\quad$ EVOLUCIÓN DE MARGEN INTENSIVO, ÍNDICE DE PRECIO E ÍNDICE DE CANTIDAD. CHINA VERSUS RESTO DEL MUNDO: 1990-2005

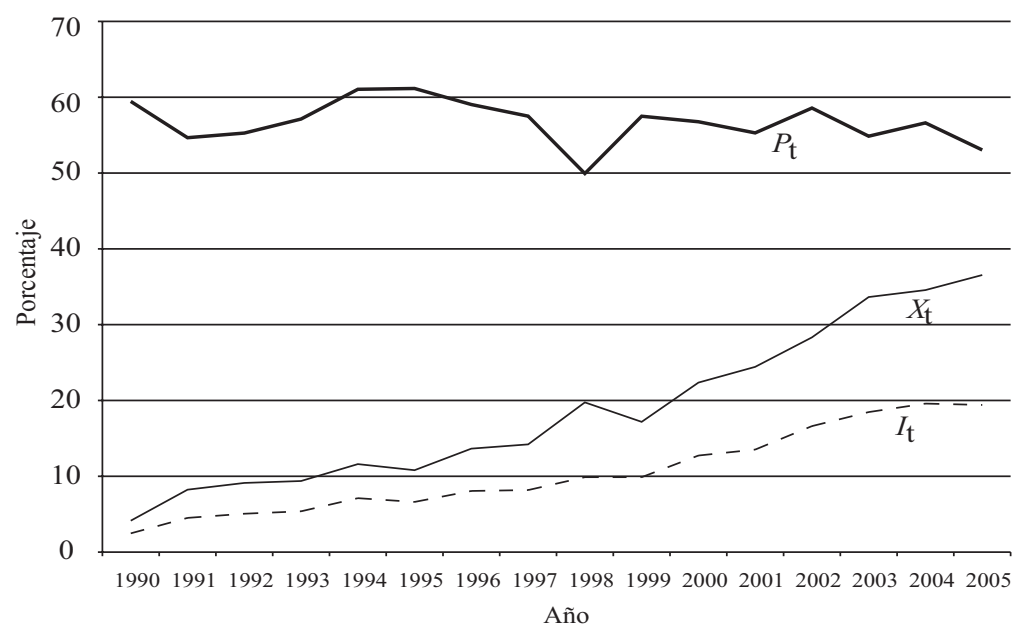

${ }^{11}$ Es importante destacar que esto es compatible con la evidencia casual de cada día que muestra una caída sostenida en los precios de los productos chinos. El índice de precios muestra el precio de los productos chinos relativo al resto del mundo, por lo que si la caída en los precios de los productos chinos genera disminuciones en los precios de los productos de otras fuentes, el índice de precios se mantiene relativamente constante. 
tos chinos sea un 36,5\% de la del resto del mundo el 2005 si los primeros cuestan la mitad? Por otra parte, el notable aumento en las cantidades importadas desde China no está acompañado de una importante caída en los precios relativos de los productos chinos. Salvo que la elasticidad-precio de demanda sea muy alta, otras variables parecen explicar el nivel y la evolución del consumo de productos chinos. Existen dos posibles explicaciones. Por un lado, aun cuando nuestro análisis se centra en productos definidos a 8 dígitos, es posible que dentro de cada producto existan distintas variedades (no observables) ofrecidas. En este caso, el mayor consumo del resto del mundo se puede deber a que ofrece un mayor número de variedades que China en un producto cualquiera. En la medida que los consumidores valoren la diversidad, esto se traducirá en mayores importaciones desde el resto del mundo aun sin diferencias internacionales en precio o calidad. Alternativamente, pueden existir diferencias en la calidad de los productos, que se traduce en diferencias en disponibilidad a pagar por variedades de distintas fuentes. En otras palabras, si los productos del resto del mundo son, en promedio, de mejor calidad que los chinos, el menor consumo de productos chinos se explica por una menor demanda.

La sección siguiente presenta un modelo simple que permite descomponer las diferencias en consumo de cualquier producto $j$ en función de diferencias en precios relativos, en calidades relativas (disposición a pagar) y en el número de variedades no observadas. De este modelo se deriva una estrategia empírica para estimar las diferencias en calidad y para descomponer la penetración de China en términos de diferencias de variedades, precio, o calidad.

\section{Modelo}

En esta sección presentamos la estructura básica del marco teórico y la estrategia empírica que de él se deriva. Para una derivación detallada del modelo, véase Álvarez y Claro (2006). Consideramos un país (Chile), representado con el supra-índice $d$ cuyos habitantes consumen distintos tipos de bienes y valoran la diversidad dentro de cada producto. Por ejemplo, los consumidores dedican un cierto porcentaje de su ingreso al consumo de zapatos, pero además valoran distintos tipos de zapatos (esto es, no les gusta ir a misa el domingo con zapatos de oficina o zapatillas de tenis). En términos de los datos usados, consideramos que cada categoría de 8 dígitos es un producto, y que cada país produce distintas variedades de cada producto. Por ejemplo, China puede producir dos tipos de zapatos mientras que Alemania puede producir siete tipos de zapatos. Definimos el consumo (en 
cantidad) en Chile de cada variedad del producto $j$ producido en el país $k$ (Chile, China o el resto del mundo) el año $t$ como $x_{k j t}^{d}$.

Cada variedad tiene una calidad distinta, que se traduce en diferentes disposiciones a pagar por parte de los consumidores. Sin embargo, la calidad está sujeta a dos restricciones. Por una parte, suponemos que todos los individuos del mundo tienen idénticas preferencias —las variedades son diferenciadas verticalmente-, lo que implica que la calidad de las variedades de un producto de un país no depende de quien las consuma. En otras palabras, suponemos que no hay diferencias en la valoración de las variedades chinas de un producto entre los consumidores japoneses y los consumidores franceses. Por otra parte, suponemos que todas las variedades de un bien producidas en un país tienen igual calidad, aun cuando ésta pueda variar entre productos. Así, todos los zapatos chinos tienen igual calidad, pero su calidad puede diferir de la calidad de los manteles chinos. De esta manera, las variedades de un bien producidas en un país son simétricas, tanto en términos de calidad como en términos de la tecnología usada para producirlas. Luego, la existencia de distintas variedades se explica por las preferencias de los individuos por distintas variedades de un mismo bien.

La demanda relativa en Chile por variedades del bien $j$ de China y del resto del mundo es:

$$
\left(\frac{x_{c j t}^{d}}{x_{r j t}^{d}}\right)=\left(\frac{p_{c j t}^{d}}{p_{r j t}^{d}}\right)^{-\sigma_{j}} \cdot\left(\frac{q_{c j t}}{q_{r j t}}\right)^{\sigma_{j}}
$$

donde $p_{c j t}^{d} / p_{r j t}^{d}$ es la razón de precio en Chile (que incluye costo de transporte) de las variedades del bien $j$ desde China y el resto del mundo y $q_{r j t}$ / $q_{c j t}$ es la razón de calidad de las variedades del bien $j$ producido en el resto del mundo y China. El parámetro $\sigma_{j}>1$ mide la elasticidad de sustitución entre variedades del mismo bien. La expresión (1) es intuitiva: el consumo en Chile es mayor de variedades con menor razón precio/calidad.

La ecuación (1) es una condición de demanda, por lo que se necesita especificar la forma de las ofertas de cada variedad para poder determinar el nivel de equilibrio de mercado para cada variedad. Para lo anterior seguimos la estructura propuesta por Krugman $(1979,1980)$, donde cada variedad se produce con funciones de retornos crecientes a escala. Esto implica que cada variedad es producida en un solo país, aun cuando los países pueden producir distintas variedades del mismo bien. De esta manera, cada produc- 
tor enfrenta una curva de demanda mundial por su variedad —que es la suma de las demandas de cada país por esa variedad-, y toma sus decisiones óptimas de precio y producción ${ }^{12}$. Suponemos que existe competencia monopolística, por lo que la libre entrada de empresas hace que en equilibrio no existan utilidades sobrenormales. La función de producción para cada variedad del bien $j$ producida en el país $k$ como $l_{k j t}=f_{j}+m_{k j t} \cdot x_{k j t}$ donde $l_{k j t}$ son los requerimientos de trabajo para producir $x_{k j t}$ unidades de una variedad del bien $j$ en el país $k$ el año $t$. El parámetro $f_{j}$ mide los requerimientos fijos de trabajo (que da origen a los retornos crecientes a escala) y $m_{k j t}$ mide los requerimientos marginales de trabajo por unidad de producto. Así, la estrategia de precio óptima de cada productor es:

$$
p_{k j t}=w_{k t} \cdot m_{k j t} \cdot\left(\sigma_{j}-1\right) / \sigma_{j}
$$

donde $w_{k t}$ es el salario en el país $k$ el año $t$, que está determinado por la tecnología en el sector de retornos constantes a escala. Es importante notar que el precio que determinan los productores está completamente determinado por condiciones de oferta - tecnología y costos laborales- y no depende del nivel de demanda mundial por cada variedad, ya que la elasticidad de sustitución entre variedades se supone constante. Así, las variedades serán más caras en aquellos países con alto salario y serán más baratas en aquellos países con mejor tecnología de producción, lo que refleja los menores requerimientos de factor por unidad de producto. Luego, los precios relativos que enfrentan los consumidores chilenos por variedades de China y el resto del mundo son:

$$
\left(\frac{p_{c j t}^{d}}{p_{r j t}^{d}}\right)=\left(\frac{w_{c t}}{w_{k t}}\right) \cdot\left(\frac{m_{c j t}}{m_{r j t}}\right) \cdot\left(\frac{\tau_{c j t}^{d}}{\tau_{r j t}^{d}}\right)
$$

donde $\tau_{k j t}^{d}>1$ mide los costos de transporte entre $k$ y $d$. Debido a que los precios enfrentados por Chile para distintas variedades no dependen de su nivel de consumo, la ecuación (1) puede ser usada para estimar una función de demanda por variedades. Debido a que no existen datos de consumo (importaciones) por variedad sino por producto (no observamos $x_{k j t}^{d}$ sino

${ }^{12}$ Suponemos que además existe un sector de retornos constantes a escala que existe en todos los países. El libre comercio de estos bienes homogéneos hace que las diferencias de salario entre países estén totalmente determinadas por diferencias entre países en la productividad en la función de producción del bien homogéneo. 
$x_{k j t}^{d}=n_{k j t} \bullet x_{k j t}^{d}$ donde $n_{k j t}$ en el número de variedades producidas en $k$ ), reescribimos (1) como:

$$
\left(\frac{X_{c j t}^{d}}{X_{r j t}^{d}}\right)=X_{j t}^{d}=\left(p_{j t}^{d}\right)^{-\sigma_{j}} \cdot\left(q_{j t}\right)^{\sigma_{j}} \cdot\left(n_{j t}\right),
$$

donde $p_{j t}=p_{c j t} / p_{r j t}, q_{j t}=q_{c j t} / q_{r j t} \mathrm{y} n_{j t}=n_{c j t} / n_{r j t}$, es la razón entre el número de variedades del bien $j$ producidos en China y el resto del mundo. Sin embargo, la cantidad de variedades de cada producto $j$ producidas por cada país no es observable. Nuestra estrategia para identificar $n_{j t}$ es resolver para la cantidad de variedades producidas en cada país consistente con el equilibrio en el mercado mundial de cada variedad y con el equilibrio en el mercado laboral de cada país ${ }^{13}$. Lo anterior permite expresar $n_{j t}$ como función de la razón del ingreso total de China y el resto del mundo $\phi_{t}=Y_{c t} / Y_{r t}$, de las diferencias en productividad $m_{j t}$, de las diferencias en calidad $q_{j t}$, de las diferencias en salarios $w_{t} \mathrm{y}$ de los costos de transporte $\tau_{j t}$ (que por simplicidad se suponen iguales entre pares de países). Así, $n_{j t}=f\left(\phi_{t}, m_{j t}\right.$, $\left.q_{j t}, w_{t}, \tau_{j t}\right)$. Las propiedades de esta función son intuitivas. Los países más grandes (con mayor ingreso total $Y$ ) atraen un mayor número de productos diferenciados porque los costos de transporten favorecen la producción en lugares cercanos a la demanda, con lo que $\partial n_{j t} / \partial \phi_{t}>0$. También, el número de variedades es mayor en países con bajos salarios, con alta productividad o en países que producen variedades de alta calidad, lo que aumenta la demanda mundial por esos productos, atrayendo a los productores de bienes diferenciados, i.e., $\partial n_{j t} / \partial w_{t}<0, \partial n_{j t} / \partial m_{j t}<0$ y $\partial n_{j t} / \partial q_{j t}>0 .{ }^{14}$ Así, tomando en cuenta que $m_{j t}=p_{j t}^{d} / w_{t}$ la ecuación (4) puede expresarse como:

$\ln X_{j t}=\alpha_{0 j}+\alpha_{1 j} \cdot \ln p_{j t}^{d}+\alpha_{2 j} \cdot \ln q_{j t}+\alpha_{3 j} \cdot \ln \phi_{t}+\alpha_{4 j} \cdot \ln w_{t}+\alpha_{5 j} \cdot \ln \tau_{j t}$

donde los coeficientes satisfacen $\alpha_{2 j}=-\left(\alpha_{1 j}+\alpha_{4 j}+1\right)$. La ecuación (5) es la base de la estimación empírica en la siguiente sección.

${ }^{13}$ Hallak y Schott (2005) suponen una relación negativa entre la razón precio/ calidad y el número de variedades exportadas por un país. Nuestro modelo endogeniza este resultado, y además incorpora el efecto del tamaño de mercado y los costos de factores en la cantidad de variedades producidas y exportadas.

${ }^{14}$ Estos resultados son consistentes con los encontrados por Romalis (2004) y Bernard et al. (2006), quienes muestran que los sectores con ventajas comparativas tienen bajos precios y un alto número de variedades. En nuestro modelo, un mayor número de variedades se debiera observar en países de bajo salario (condicional al ingreso total) y en países de alta productividad. Ambas condiciones generan bajos precios de los productos. 


\section{Estimación Empírica}

Todas las variables de la ecuación son observables con la excepción de la calidad relativa. Suponemos que la razón de calidad de las variedades de China y el resto del mundo tiene la siguiente forma funcional: $\ln q_{j t}=\delta_{0 j}$ $+\delta_{1 j} \bullet t+\delta_{2 j} \bullet t^{2}$, donde $t$ es una variable de tendencia. Esto significa que la calidad tiene un componente específico en cada producto, y una tendencia no lineal que también es específica en cada producto. Por lo tanto el panel a estimar es el siguiente:

$\ln X_{j t}=\beta_{0 j}+\beta_{1 j} \cdot \ln p_{j t}^{d}+\beta_{2 j} \cdot t+\beta_{3 j} \cdot t^{2}+\beta_{4 j} \cdot \ln \phi_{t}+\beta_{5 j} \cdot \ln w_{t}+\beta_{6 j} \cdot \ln \tau_{j t}+\mu_{j t}$

Empíricamente, $p_{j t}^{d}$ es calculado como la razón de precios (valores unitarios) CIF de las importaciones de China y el resto del mundo en cada categoría de 8 dígitos. $\phi_{t}$ es calculado como la razón del producto interno bruto (PPP) de China y el resto del mundo, $w_{t}$ es la razón de ingreso per cápita (PPP) entre China y el resto del mundo. Finalmente, controlamos por la evolución de las tarifas efectivas promedio de Chile como medida de $\tau_{j t}$. La tasa de crecimiento de la razón de calidad $q_{j t}$ es igual a $\delta_{1 j}+2 \bullet \delta_{2 j} \bullet t$, que se obtiene calculando la distribución de

$$
\delta_{1 j}=-\beta_{2 j} /\left(\beta_{1 j}+\beta_{5 j}+1\right) \text { y } \delta_{2 j}=-\beta_{3 j} /\left(\beta_{1 j}+\beta_{5 j}+1\right) .
$$

El Cuadro $\mathrm{N}^{\circ} 3$ muestra las estimaciones de la tasa de crecimiento promedio anual de la razón de calidad $q_{j t}$ suponiendo que la tasa de crecimiento en la calidad relativa es la misma en todos los productos. Esto es, suprimimos el sub-índice $j$ en los coeficientes de la ecuación (6) y estimamos coeficientes comunes para todos los productos. Para evitar problemas derivados de errores de digitación en Aduanas, presentamos las estimaciones excluyendo de la muestra aquellas observaciones con datos de precio relativo muy bajas o muy altas. Los resultados son robustos a distintos tamaños de la muestra. En promedio, la tasa de crecimiento de la calidad (disposición a pagar) de las variedades chinas es un $0,8 \%$ superior a la de las variedades del resto del mundo, y la tasa de crecimiento de la calidad relativa de las variedades chinas es creciente. Al final de la muestra, la tasa de crecimiento en la calidad relativa de los productos chinos es superior al $1 \%$ anual.

Como se deriva del Cuadro $\mathrm{N}^{\circ}$ 2, la tasa de crecimiento promedio anual del margen intensivo $I_{t}$ es algo inferior al 15\%. La contribución al crecimiento $I_{t}$ de cambios en precios relativos de productos similares es 
CUADRO N ${ }^{\circ}$ 3: $\quad$ TASA DE CRECIMIENTO ANUAL DE LA RAZÓN DE CALIDAD CHINA/RESTO DEL MUNDO $Q_{j t}$

1990-2005

\begin{tabular}{|c|c|c|c|c|c|c|}
\hline \multirow{3}{*}{$\begin{array}{l}\text { Año } \\
1990\end{array}$} & \multicolumn{3}{|c|}{$0.05<P_{j t}<20$} & \multicolumn{3}{|c|}{$0.1<P_{j t}<10$} \\
\hline & \multirow{2}{*}{$\begin{array}{c}\text { Tasa }(\%)^{\mathrm{b}} \\
0,1\end{array}$} & \multicolumn{2}{|c|}{$90 \%$ intervalo de Conf. } & \multirow{2}{*}{$\begin{array}{r}\text { Tasa }(\%)^{\mathrm{b}} \\
0,1\end{array}$} & \multicolumn{2}{|c|}{$90 \%$ intervalo de Conf. } \\
\hline & & $-0,2$ & 0,3 & & $-0,3$ & 0,4 \\
\hline 1991 & 0,2 & $-0,1$ & 0,4 & 0,1 & $-0,1$ & 0,4 \\
\hline 1992 & 0,3 & 0,0 & 0,5 & 0,2 & 0,0 & 0,5 \\
\hline 1993 & 0,3 & 0,1 & 0,6 & 0,3 & 0,1 & 0,6 \\
\hline 1994 & 0,4 & 0,1 & 0,7 & 0,4 & 0,1 & 0,7 \\
\hline 1995 & 0,5 & 0,2 & 0,9 & 0,5 & 0,1 & 0,9 \\
\hline 1996 & 0,6 & 0,2 & 1,0 & 0,6 & 0,2 & 1,0 \\
\hline 1997 & 0,7 & 0,2 & 1,2 & 0,7 & 0,2 & 1,2 \\
\hline 1998 & 0,8 & 0,2 & 1,3 & 0,8 & 0,2 & 1,4 \\
\hline 1999 & 0,9 & 0,2 & 1,5 & 0,9 & 0,2 & 1,5 \\
\hline 2000 & 0,9 & 0,3 & 1,6 & 1,0 & 0,2 & 1,7 \\
\hline 2001 & 1,0 & 0,3 & 1,8 & 1,0 & 0,2 & 1,9 \\
\hline 2002 & 1,1 & 0,3 & 1,9 & 1,1 & 0,2 & 2,1 \\
\hline 2003 & 1,2 & 0,3 & 2,1 & 1,2 & 0,2 & 2,3 \\
\hline 2004 & 1,3 & 0,3 & 2,2 & 1,3 & 0,2 & 2,4 \\
\hline 2005 & 1,4 & 0,3 & 2,4 & 1,4 & 0,2 & 2,6 \\
\hline Promedio $^{a}$ & 0,8 & 0,2 & 1,3 & 0,8 & 0,2 & 1,4 \\
\hline
\end{tabular}

Notas:

Columna $0.05<p_{j t}<20$ considera estimaciones donde se excluyen las observaciones producto-año donde la razón de precio entre el producto chino y el producto promedio del resto del mundo en cada categoría 8 dígitos es inferior a $5 \%$ y superior a 20. Similar criterio se aplica en la columna $0.1<p_{j t}<10$.
a: Tasa de crecimiento promedio anual compuesta
b: Tasa de crecimiento anual
c: Intervalo de confianza al $90 \%$

$\left(1+\beta_{1}\right) \cdot d \ln P_{t}$ donde $d \ln P_{t}$ es el cambio porcentual observado en los precios relativos de los productos chinos vis-à-vis el resto del mundo. De acuerdo a los resultados obtenidos, la caída en el precio relativo de los productos chinos explica un crecimiento anual del margen intensivo entre 2 y 6 puntos porcentuales. La contribución de las mejoras en calidad de los productos chinos - tanto por su efecto directo en mayor demanda de cada variedad como por su efecto indirecto a través de un mayor número de variedades producidas - es $\left(\beta_{2}+2 \cdot \beta_{3} \bullet t\right)$. Las mejoras en calidad explican cerca de 20 puntos porcentuales de crecimiento en la penetración de productos chinos. El resto del crecimiento en el Margen Intensivo se explica 
por cambios en el tamaño de la economía china y por el crecimiento en sus costos de producción.

Las estimaciones anteriores imponen una tasa de crecimiento en la calidad promedio de los productos chinos igual para todas las categorías de 8 dígitos. Relajamos esta restricción permitiendo que los coeficientes de la regresión difieran de acuerdo con la elasticidad de sustitución entre variedades de cada producto. Tomamos los valores de $\sigma_{j}$ estimados por Broda y Weinstein (2006) e interactuamos las variables independientes en (6) con una variable dummy que toma valor 1 si $\sigma_{j}$ es mayor que la media muestral (3.4) y 0 si $\sigma_{j}$ es menor que la media. Así, estimamos la tasa de crecimiento promedio de la calidad de las variedades chinas respecto a las variedades del resto del mundo para un subconjunto de productos diferenciados (bajo $\sigma_{j}$ ) y para un subconjunto de productos homogéneos (alto $\sigma_{j}$ ). Los resultados se presentan en el Cuadro $\mathrm{N}^{\circ} 4$. La tasa de crecimiento de la calidad de las variedades chinas es significativamente mayor al resto del mundo en productos diferenciados, mientras que en productos homogéneos no se observan cambios significativos en la razón de calidad. En otras palabras, la mejora en calidad de las variedades chinas en comparación con las del resto del mundo es mayor en productos diferenciados, mientras que no hay evidencia de cambios significativos en la calidad relativa en productos homogéneos.

La Figura No 3 muestra la tasa de crecimiento promedio anual de la razón de precios entre variedades de China y el resto del mundo para productos con distinta elasticidad de sustitución ${ }^{15}$. La caída en el precio relativo de las variedades es evidente en productos altamente diferenciados, lo que coincide con una mejora en su calidad relativa. En cambio, en productos homogéneos observamos convergencia en precios relativos sin cambios en la calidad ${ }^{16}$. De acuerdo con el modelo, los precios de las variedades chinas reflejan los precios de los factores en China y su productividad. De esta manera, el crecimiento en los precios de los factores en China —reflejados en la convergencia del ingreso per cápita con respecto al resto del mundo - tiende a hacer aumentar los precios relativos de los productos chinos en relación con el resto del mundo ${ }^{17}$. La evidencia sugie-

${ }^{15}$ Esta Figura está basada en los resultados de la regresión $\ln p_{j t}=\gamma_{0 j}+\gamma_{1} t+$ $\gamma_{2} \sigma_{j}+\gamma_{3} \sigma_{j} t+\zeta_{j t}$

${ }_{16}$ También es cierto que el aumento en la penetración de productos chinos ha sido mayor en productos altamente diferenciados.

${ }^{17}$ Por supuesto, el impacto sobre los costos marginales de producción de los cambios en precios de factores dependerá de la participación de los distintos factores en los costos de cada producto — elemento que está ausente del análisis debido al supuesto que trabajo es el único factor de producción. 
CUADRO No 4: TASA DE CRECIMIENTO ANUAL DE LA RAZÓN DE CALIDAD CHINA/RESTO DEL MUNDO $Q_{j t}$ PRODUCTOS DIFERENCIADOS VERSUS PRODUCTOS HOMOGÉNEOS $0.05<p_{j t}<20$

\begin{tabular}{|c|c|c|c|c|c|c|}
\hline \multirow{3}{*}{$\begin{array}{l}\text { Año } \\
1990\end{array}$} & \multicolumn{3}{|c|}{$\begin{array}{l}\text { Productos diferenciados } \\
\text { (bajo } \sigma_{j} \text { ) }\end{array}$} & \multicolumn{3}{|c|}{$\begin{array}{l}\text { Productos homogéneos } \\
\text { (alto } \sigma_{j} \text { ) }\end{array}$} \\
\hline & \multirow{2}{*}{$\begin{array}{c}\text { Tasa }(\%) \\
0,1\end{array}$} & \multicolumn{2}{|c|}{$\begin{array}{l}90 \% \text { intervalo } \\
\text { de Conf. }\end{array}$} & \multirow{2}{*}{$\begin{array}{c}\text { Tasa }(\%) \\
0,1\end{array}$} & \multicolumn{2}{|c|}{$\begin{array}{l}90 \% \text { intervalo } \\
\text { de Conf. }\end{array}$} \\
\hline & & $-0,2$ & 0,4 & & $-0,4$ & 0,6 \\
\hline 1991 & 0,2 & $-0,1$ & 0,4 & 0,2 & $-0,3$ & 0,7 \\
\hline 1992 & 0,2 & 0,0 & 0,5 & 0,3 & $-0,2$ & 0,9 \\
\hline 1993 & 0,3 & 0,1 & 0,6 & 0,4 & $-0,2$ & 1,0 \\
\hline 1994 & 0,4 & 0,1 & 0,7 & 0,5 & $-0,3$ & 1,2 \\
\hline 1995 & 0,5 & 0,1 & 0,9 & 0,6 & $-0,3$ & 1,4 \\
\hline 1996 & 0,6 & 0,2 & 1,0 & 0,6 & $-0,3$ & 1,6 \\
\hline 1997 & 0,7 & 0,2 & 1,2 & 0,7 & $-0,4$ & 1,8 \\
\hline 1998 & 0,8 & 0,2 & 1,3 & 0,8 & $-0,4$ & 2,0 \\
\hline 1999 & 0,8 & 0,2 & 1,5 & 0,9 & $-0,5$ & 2,2 \\
\hline 2000 & 0,9 & 0,2 & 1,7 & 1,0 & $-0,5$ & 2,5 \\
\hline 2001 & 1,0 & 0,2 & 1,8 & 1,0 & $-0,6$ & 2,7 \\
\hline 2002 & 1,1 & 0,2 & 2,0 & 1,1 & $-0,7$ & 2,9 \\
\hline 2003 & 1,2 & 0,2 & 2,2 & 1,2 & $-0,7$ & 3,1 \\
\hline 2004 & 1,3 & 0,2 & 2,3 & 1,3 & $-0,8$ & 3,4 \\
\hline 2005 & 1,4 & 0,2 & 2,5 & 1,4 & $-0,8$ & 3,6 \\
\hline $\begin{array}{l}\text { Tasa compuesta de } \\
\text { crecimiento anual }\end{array}$ & 0,8 & 0,2 & 1,3 & 0,8 & $-0,4$ & 2,0 \\
\hline
\end{tabular}

Notas:

Productos diferenciados se refiere a productos (categorías de 8 dígitos sistema armonizado) con elasticidad de sustitución entre variedades $s_{j}$ menor que 3.4 (media). Productos homogéneos incluye productos con elasticidad de sustitución mayor que 3.4.

re que en productos diferenciados estas alzas en costos de factores han sido compensadas por mejoras en productividad, impidiendo un aumento en los precios de las variedades producidas en China. Esto coincide con las mejoras en calidad de las variedades chinas en estos productos, lo que es consistente con la idea que la mayor disposición a pagar por productos chinos está directamente relacionada con mejoras en su tecnología de producción. Las mejoras en productividad en productos diferenciados en China han permitido mantener precios bajos — compensando los aumentos en costos de factores-, aumentando la disposición a pagar por sus variedades e incentivando la producción de un mayor número de variedades. En productos homogéneos, el alza en precios relativos de las variedades chinas 
FIGURA N ${ }^{\circ}$ 3: CRECIMIENTO PROMEDIO ANUAL DE PRECIO RELATIVO CHINA/RESTO DEL MUNDO POR PRODUCTO.

TASA PROMEDIO COMPUESTA ANUAL E INTERVALO DE CONFIANZA AL $90 \%$

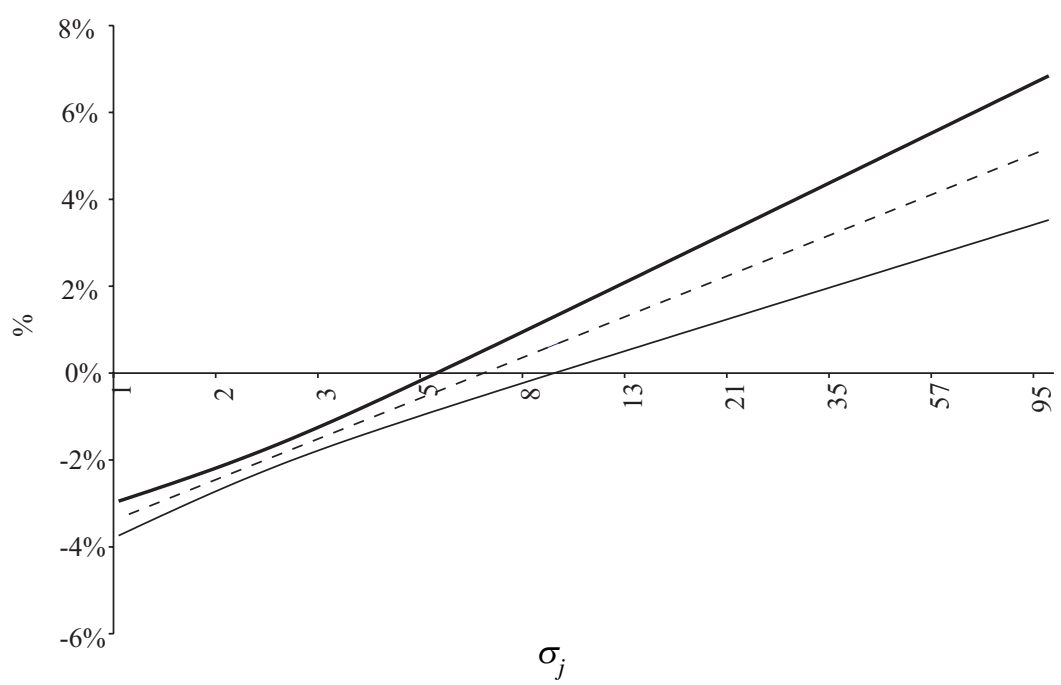

sugiere que las ganancias en productividad han sido menores, lo que coincide con una razón de calidad China/resto del mundo constante ${ }^{18}$.

Un estudio de las causas de las mejoras en productividad en las industrias productoras de bienes diferenciados en China escapa a los objetivos de este trabajo. Sin embargo, nuestra conjetura es que la penetración de empresas extranjeras explica un porcentaje importante de las mejoras en productividad en China. Esto se basa en conclusiones obtenidas en otros trabajos. Claro (2006b) estudia las diferencias en rentabilidad del capital y productividad entre empresas extranjeras y empresas domésticas en China el año 2003 y muestra que las segundas tienen mayor productividad en aquellos sectores donde las empresas extranjeras son dominantes, mientras que la desventaja de las empresas domésticas es mayor en aquellos sectores donde la participación de las empresas extranjeras es baja. Ya sea porque la presencia de empresas extranjeras impulsa la productividad doméstica o porque las autoridades chinas restringen la producción de empresas extranjeras en sectores donde la productividad doméstica es muy baja, existe una correlación positiva entre productividad en China — tanto de empre(2006).

${ }^{18}$ Un análisis más profundo de estos resultados se encuentra en Álvarez y Claro 
sas domésticas como extranjeras-y la participación de empresas extranjeras en la producción total. Asimismo, Brambilla (2006) muestra que las empresas extranjeras en China, que tienen mayor productividad que las domésticas, introducen un mayor número de variedades.

\section{Conclusiones}

Los productos chinos son, en promedio, significativamente más baratos que productos similares del resto del mundo. Sin embargo, siempre lo han sido, y entre 1990 y 2005 se observa sólo una pequeña tendencia al aumento en esta brecha. Luego, no son cambios en los precios relativos lo que explica la alta tasa de crecimiento de las importaciones desde China. En este trabajo presentamos evidencia de que el principal motor de la penetración de China es un incremento en la calidad de sus variedades. La mayor disposición a pagar no sólo ha aumentado la demanda por variedades chinas - lo que explica un mayor consumo sin cambios en precios relativossino que también ha incrementado fuertemente la disponibilidad de variedades desde China. En otras palabras, después de 15 años de fuerte apertura al comercio exterior en China concluimos que la principal causa de sus mayores exportaciones son la mayor calidad de sus productos y la mayor disponibilidad de variedades.

Los resultados sugieren que este fenómeno está impulsado por un fuerte aumento en la productividad de las empresas en China, ya sea por reformas a las empresas domésticas o por la llegada de empresas de propiedad extranjera. Esta conclusión está basada en dos resultados obtenidos. Por un parte, no se observa un aumento en los precios relativos de las variedades chinas — sino más bien una caída - en un período con clara convergencia en los precios de los factores. Esto podría estar explicado por incrementos en la productividad de las empresas chinas que impiden que los aumentos en costos de factores se trasladen a precios. Por otra parte, la mayor caída en precios de variedades chinas se observa en productos altamente diferenciados, que son aquellos con mayor aumento en la disposición a pagar por variedades chinas. Por otra parte, en productos homogéneos no observamos cambios significativos en la calidad relativa de las variedades, lo que es consistente con la convergencia observada en precios relativos. Luego, la mayor productividad en China ha permitido mantener los precios bajos, aumentar la calidad de los productos y aumentar el número de variedades ofrecidas desde China.

Estos resultados tienen importantes implicancias para el análisis de los efectos de la competencia china en un país como Chile. La capacidad de 
diferenciar los productos manufactureros por calidad depende de la capacidad de afectar la productividad como lo sugiere la experiencia de China. Sin reformas que incentiven fuertemente las mejoras en productividad o que permitan asimilar mejor los adelantos tecnológicos en el mundo entero - como una reforma educacional de fondo - resulta difícil imaginar que los países pequeños serán capaces de competir con un gigante como China en los mercados mundiales de productos manufactureros. El caso de Estados Unidos es clarificador en este sentido. Su ventaja comparativa no está en la manufactura propiamente tal sino en su capacidad de desarrollar nueva tecnología y productos de alta calidad que son manufacturados en China, por ejemplo, iPod. Esta ventaja radica principalmente en su fuerza de trabajo altamente creativa y con elevado capital humano. El caso de los países escandinavos no es muy diferente. En la medida en que Chile mantenga una fuerza laboral con baja capacitación la posibilidad de desarrollar productos diferenciados de alta calidad es muy pequeña, y su capacidad de producirlos también es pequeña debido a los menores costos de producción en países como China. La consecuencia natural de este proceso es una tendencia a la producción de bienes intensivos en recursos naturales, donde las ventajas comparativas de países como Chile son evidentes.

\section{REFERENCIAS}

Abreu, M.: (2006) “China’s Emergence in the Global Economy and Brazil”. En Integration and Trade Journal, $\mathrm{N}^{\circ}$ 24: 123-152.

Adams, G., B. Gangnes, y Y. Shachmurove: (2004) "Why is China so Competitive? Measuring and Explaining China’s Competitiveness”. Working Paper 07-2004, Singapore Management University.

Álvarez, R. y S. Claro: (2006) "The China Phenomenon: Price, Quality or Variety”. Mimeo, Instituto de Economía, Pontificia Universidad Católica de Chile.

Arellano, R.: (2006) "Implications of China's Emergence in the Global Economy for Latin America: The Case of Mexico". En Integration and Trade Journal, $\mathrm{N}^{\circ}$ 24: 213-250.

Bernard, A., B. Jensen y P. Schott: (2006) "Survival of the Best Fit: Exposure to LowWage Countries and the (Uneven) Growth of US Manufacturing Plants”. En Journal of International Economics, $\mathrm{N}^{\circ}$ 68: 219-237.

Bernard, A., S. Redding y P. Schott: "Heterogeneous Firms and Comparative Advantage”. Por aparecer en Review of Economic Studies.

Beyer, H., P. Rojas y R. Vergara: (2000) “Apertura Comercial y Desigualdad Salarial en Chile”. En Estudios Públicos, N 77.

Brambilla, Irene: (2006) "Multinationals, Technology, and the Introduction of Varieties of Goods”. NBER Working Paper No 12217.

Broda, C. y D. Weistein: (2006) "Globalization and the Gains from Varieties". En Quarterly Journal of Economics, 121 (2): 541-585 
Claro, S.: (2006a) "Implications of China's Emergence in the Global Economy for Latin America and the Caribbean Region: The Case of Chile”. En Integration and Trade Journal, $\mathrm{N}^{\circ}$ 24: 153-212.

(2006b) "Heterogeneous Performance in China's Manufacturing Sector”. Mimeo, Instituto de Economía, Pontificia Universidad Católica de Chile.

Dussel, E. y L. X. Dong: (2004) “Oportunidades y Retos Económicos de China para México y Centroamérica”. CEPAL, México.

Feenstra, R.: (1994) "New Product Varieties and the Measurement of International Prices”. En American Economic Review, 84 (1): 157-177.

Galperín, C., G. Girado y E. Rodríguez Diez: (2006) “Consequences of China’s New Role in the International Economy for Latin America: The Argentine Case”. En Integration and Trade Journal, $\mathrm{N}^{\circ}$ 24: 89-121.

Hallak, J. C. y P. Schott: (2005) "Estimating Cross-country Differences in Product Quality”. Mimeo, Yale School of Management.

Hanson, G. y R. Robertson: (2006) “The Evolution of Mexico’s Manufacturing Exports: A Gravity Framework for Evaluating Supply and Demand Factors”. Artículo presentado en Public Finance-Labor Economics Seminar, U.C. Davis, abril.

Hummels, D. y P. Klenow: (2005) “The Variety and Quality of a Nation's Exports”. En American Economic Review, No 95: 704-723.

Krugman, P.: (1979) "Increasing Returns, Monopolistic Competition and International Trade". En Journal of International Economics, 9 (4): 469-479. (1980) "Scale Economies, Product Differentiation and the Pattern of Trade”. En American Economic Review, 70 (5): 950-959.

Leamer, E.: (2006) “A Flat World, A Level Playing Field, a Small World After All, or None of the Above?” En Journal of Economic Literature, por aparecer.

Leamer, E., H. Maul, S. Rodriguez y P. Schott: (1999) "Does Natural Resource Abundance Cause Latin American Income Inequality?” En Journal of Development Economics, 59 (1): 3-42

Lederman, D. y W. Maloney: (2002) “Open Questions about the Link between Natural Resources and Economic Growth: Sachs and Warner Revisited”. Mimeo, Washington D.C., Banco Mundial.

Manzano, O. y R. Rigobon: (2001) "Resource Curse or Debt Overhang”. NBER Working Paper $\mathrm{N}^{\circ} 8390$.

Rodrik, D.: (2006) “What’s So Special About China’s Exports?” Working Paper No 06001, John Kennedy School of Government, Harvard University.

Romalis, J.: (2004) "Factor Proportions and the Structure of Commodity Trade". En American Economic Review, № 94: 67-97.

Sachs, J. y A. Warner: (1997) "Sources of Slow Growth in African Economies”. En Journal of African Economies, 6 (3): 335-376.

Sato, K.: (1976) “The Ideal Log-Change Index Number”. En The Review of Economics and Statistics, 58 (2): 223-228.

Schott, P.: (2004) “Across-Product versus Within-Product Specialization in International Trade”. En Quarterly Journal of Economics, 119 (2): 647-678.

(2006) “The Relative Sophistication of Chinese Exports”. NBER Working Paper $N^{\circ} 12173$. 